\title{
EDITORIAL
}

\section{Special Issue on Cognitive Disorders}

DOI:http://dx.doi.org/10.5770/cgj.15.61

The editorial board of the Canadian Geriatrics Journal would like to present our last collection of articles for 2012, an issue devoted entirely to memory disorders. In this special publication (jointly with the Canadian Journal of Neurological Sciences), we are pleased to provide the latest recommendations from the fourth Canadian Consensus Conference on the Diagnosis and Treatment of Dementia. These latest guidelines address many issues, including the early diagnosis of Alzheimer's disease using neuro-imaging/biomarkers, and the management of rapidly progressive and early onset dementia.

In keeping with the theme of memory disorders, Dr. Azad from the University of Ottawa performed a feasibility study of the use of a telemedicine clinic to diagnose dementia in a rural setting. Once the diagnosis of dementia is made, however, two of the most common complications include issues with driving and issues with maintaining activities of daily living. Dr. Marshall addresses the first issue by examining the current practice of various medical specialists in assessing driving fitness in the setting of dementia. Cheryl Cook and Ken Rockwood from the University of Dalhousie address the second issue in a novel way-examining, through metaethnography, the perspectives of paid dementia care workers on their jobs.

I hope you enjoy our special (and final issue) for 2012!

Cheers,

Dr. Kenneth M. Madden

Editor-in-Chief Canadian Geriatrics Journal 